Bundesgesundheitsbl 2020 $63: 749-760$ https://doi.org/10.1007/s00103-020-03152-5 Online publiziert: 28. Mai 2020

(C) Der/die Autor(en) 2020

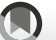

Check for
updates

Bundesverband Deutscher Krankenhausapotheker e.V. (ADKA) - Deutsche Gesellschaft für Hygiene und Mikrobiologie e.V. (DGHM) - Deutsche Gesellschaft für Infektiologie e.V. (DGI) · Deutsche Gesellschaft für Krankenhaushygiene e.V. (DGKH) · Deutsche Gesellschaft für Pädiatrische Infektiologie e. V. (DGPI) · Kommission Antiinfektiva, Resistenz und Therapie (Kommission ART) beim Robert Koch-Institut · Paul-Ehrlich-Gesellschaft für Chemotherapie e. V. (PEG) • Robert Koch-Institut, Geschäftsstelle der Kommission ART ${ }^{1}$

' Geschäftsstelle der Kommission ART 37 „Nosokomiale Infektionen, Surveillance von Antibiotikaresistenz und -verbrauch, Abteilung für Infektionsepidemiologie", Robert Koch-Institut, Berlin, Deutschland

\title{
Strukturelle und personelle Voraussetzungen für die Sicherung einer rationalen Antiinfektivaverordnung in Krankenhäusern
}

\section{Positionspapier der Kommission Antiinfektiva, Resistenz und Therapie (Kommission ART) beim Robert Koch-Institut mit Beratung durch Fachgesellschaften}

\section{Hintergrund}

Das gesteigerte Bewusstsein für die Problematik zunehmender Multiresistenzen und der drohende Verlust geeigneter Therapieoptionen für Infektionskrankheiten haben Veränderungen der Rahmenbedingungen in der Patientenversorgung zugunsten wirksamer Maßnahmen zur Eindämmung von Resistenzentwicklung und -verbreitung ermöglicht. Die gesetzlichen Grundlagen zur Umsetzung von Hygienemaßnahmen wurden verbessert. Die Sicherstellung einer rationalen Antiinfektivaverordnung mit dem Ziel einer Resistenzminimierung wird in vielen nationalen Programmen für alle Altersgruppen und Fachbereiche/Disziplinen gefordert (Antibiotic Stewardship, abgekürzt ABS). Entsprechende Maßnahmen und Instrumente wurden in mehreren Ländern in gesundheitspolitischen Empfehlungen und Leitlinien der Fachgesellschaften konkretisiert [1-11].
Mit ABS sind koordinierte, interdisziplinär abgestimmte Maßnahmen gemeint, die den Einsatz von Antiinfektiva in Krankenhäusern und anderen Gesundheitseinrichtungen, unter Berücksichtigung wissenschaftlicher Evidenz sowie von Daten zu Antibiotikaverbrauch und -resistenz, nachhaltig verbessern sollen [10-13]. In Deutschland und anderen Ländern wird seit einigen Jahren spezielle Fortbildung zu ABS von Fachgesellschaften und Ärztekammern angeboten [14]. ABS ist eine Qualifikation, die im Kontext der Infektionsmedizin den speziellen Aspekt eines verbesserten Antiinfektivaeinsatzes strukturiert und systematisch in die infektiologische Patientenversorgung integriert. ABS ist somit ein Bereich der Infektionsmedizin und eine wesentliche Komponente der Qualitätssicherung, ersetzt aber weder Infektiologie noch Mikrobiologie oder Hygiene. Die Ziele von ABS betreffen nicht nur den Krankenhausbereich, sondern ebenso die ambulante Humanmedizin und die Veterinärmedizin, in denen ein weiterer, großer Teil der Verordnung von Antiinfektiva erfolgt. Die Überlegungen in diesem Positionspapier beziehen sich jedoch nur auf den stationären Bereich. Für den ambulanten Bereich und die Veterinärmedizin sind eigene ABS-Strategien und -Methoden erforderlich.

\section{Spezielle Fortbildung und} Weiterbildung

Die Zukunft der Fortbildung und Qualifizierung zu ABS kann ohne eine generelle Verständigung über die Zukunft der Infektionsmedizin in Deutschland und damit verbundene Regelungen für die Weiterbildung nicht adäquat gesteuert werden. $[15,16]$. Der nicht unmittelbar klinisch tätige Arzt (Facharzt für Mikrobiologie, Virologie und Infektionsepidemiologie, Facharzt für Hygiene und Umwelt- 
medizin oder Facharzt mit Zusatzweiterbildung Krankenhaushygiene oder mit abgeschlossener strukturierter curricularer Fortbildung Krankenhaushygiene) bzw. Apotheker kann durch ABS-Expertise im Team dazu beitragen, dass sich die Antiinfektivastrategien verbessern. Ein relevanter Einfluss auf die individuelle komplexe infektionsmedizinische Patientenversorgung wird jedoch ohne eine gleichzeitige Stärkung der Infektiologie/ Infektionsmedizin nicht substanziell gelingen.

Es wird durch die in der neuen (Muster-)Weiterbildungsordnung 2018 für Ärzte verankerte Zusatz-Weiterbildung Infektiologie in einem Gebiet der unmittelbaren Patientenversorgung oder in Mikrobiologie, Virologie und Infektionsepidemiologie oder in Hygiene und Umweltmedizin und durch die beantragte Implementierung der Infektiologie als eigenständige Facharztkompetenz im Gebiet innere Medizin (bislang lediglich in wenigen Landesärztekammern bestehend) zu einer bedeutsamen Aufwertung infektiologischer Qualifikation kommen. ABS als Teilqualifikation mit spezifischen interventionellen Inhalten und Methoden (Fallreviews, Punktprävalenzerhebungen, Audits, Feedbackverfahren) ist als eigener Weiterbildungsblock in den Inhalten der Zusatz-Weiterbildung und der geplanten Facharzt-Weiterbildung Infektiologie verankert. Damit ist die gegenwärtige Fortbildung zum ABSExperten als Zwischenlösung zu verstehen. Bei den Apothekern erfolgte schon 2015 die Aufwertung infektiologischer Qualifikation durch die Etablierung der Bereichsweiterbildung Infektiologie, welche ABS als Bestandteil der Weiterbildung ebenfalls bereits enthält.

\section{Gesetzliche Regelungen und evidenzbasierte Leitlinien}

In Deutschland ist ABS im Krankenhaus unter Berücksichtigung von Verbrauchszahlen und Antibiotikaresistenz in Verbindung mit einer entsprechenden Qualitäts- und Qualifizierungsinitiative im Infektionsschutzgesetz $\$ 23$ gesetzlich verankert worden $[12,13$, 17]. Die Fachgesellschaften in Deutschland haben Empfehlungen für $\mathrm{ABS}$ in
Akutkrankenhäusern in Form der S3Leitlinie „Strategien zur Sicherung einer rationalen Antibiotika-Anwendung im Krankenhaus" sowie in der ergänzenden S2k-Leitlinie „Antibiotic Stewardship Konzeption und Umsetzung in der stationären Kinderheilkunde" formuliert $[9,18]$. Diese stützen sich auf internationale Leitlinien und Studien $[2,4,7$, 19-27], die nach Anwendung von ABSMaßnahmen bessere Behandlungserfolge, eine erhöhte Patientensicherheit und Minimierung unerwünschter Arzneimittelwirkungen in allen Altersgruppen zeigen konnten. Als weitere Erfolge konnten über die Implementierung von ABSMaßnahmen durch verkürzte Liegedauer und Reduktion eines übermäßigen Antiinfektivaeinsatzes auch Kostensenkungen und nicht zuletzt die angestrebte Eindämmung von Resistenzen und die Minimierung der Selektion von Clostridioides difficile belegt werden [21-31].

\section{Geschultes Personal und ABS- Teams}

Der Versuch, verschiedene Maßnahmen zum verbesserten Antiinfektivaeinsatz strukturiert und systematisch in die konkrete Patientenversorgung $\mathrm{zu}$ integrieren, verlangt Infektiologen bzw. ABS-geschultes Fachpersonal vor Ort. $\mathrm{Zu}$ den wichtigsten Voraussetzungen für effektive ABS-Maßnahmen gehören damit personelle Ressourcen. In Übereinstimmung mit Recherchen und Leitlinien aus dem In- und Ausland werden im vorliegenden Positionspapier ABS-Teams auch für deutsche Krankenhäuser gefordert. Im Folgenden werden ihre Aufgaben und ihre personelle und strukturelle Absicherung unter den hiesigen Rahmenbedingungen beschrieben.

Mandatierung und Unterstützung durch die Krankenhausleitung sowie die Bereitstellung ausreichender Ressourcen sind unabdingbare Voraussetzung für die erfolgreiche Arbeit des ABS-Teams.

\section{Aufgaben und Qualifikationen}

Die Krankenhausleitungen sollten ein ABS-Team berufen und in den klinischen Fachabteilungen/Fachabteilungsgruppen sollten ABS-beauftragte Ärzte als An- sprechpartner benannt werden. Das ABS-Team soll eine Geschäftsordnung haben, in der die Verantwortlichkeiten sowie die Zusammenarbeit mit klinischen Fachabteilungen oder deren Delegierten und den Krankenhauskommissionen geregelt sind.

\section{Aufgaben des ABS-Teams}

Das ABS-Team soll krankenhausweit und abteilungsübergreifend arbeiten. Seine Hauptaufgabe besteht in der Ausarbeitung und Umsetzung eines auf die lokalen Bedürfnisse, die Möglichkeiten und die Situation des Krankenhauses zugeschnittenen ABS-Programms unter Berücksichtigung von Daten zum Antiinfektivaeinsatz, zu Infektionserregern und zur Resistenz. Dazu gehören u.a. auch die Erstellung und Implementierung hausinterner Empfehlungen und Behandlungspfade zur Diagnostik, Prophylaxe und Therapie der wichtigsten Infektionskrankheiten. Eine Zusammenfassung der Aufgaben enthält $\bullet$ Infobox 1 .

Eine enge Kooperation mit der Krankenhausleitung, den klinischen Fachabteilungen und den dort benannten ABSbeauftragten Ärzten ist wesentlich und fördert die Akzeptanz aller Krankenhausmitarbeiter gegenüber dem ABSProgramm und dessen Erfolg. Das ABSTeam soll in der Arzneimittel- und Hygienekommission vertreten sein, jedoch eine eigene Antiinfektiva- oder ABS-Kommission etablieren. Eine Kooperation, beispielsweise in Form einer gemeinsamen Infektions- und Hygienekommission, kann je nach lokalen Gegebenheiten sinnvoll sein. Die Aufgaben des ABS-Teams können durch eine klinische Abteilung oder zentrale Einrichtung Infektiologie in Zusammenarbeit mit der Mikrobiologie, Krankenhaushygiene und Apotheke übernommen werden. Im Bereich ABS sollen zusätzlich zum Bereich Krankenhaushygiene eigenständige Strukturen etabliert sein und eng miteinander kooperieren. Krankenhaushygiene und ABS sind nicht deckungsgleich und können sich gegenseitig nicht ersetzen, sie ergänzen sich vielmehr in ihren präventiven Zielsetzungen. Neben überlappenden Themenfeldern haben beide Bereiche 
Bundesgesundheitsbl 2020 63:749-760 https://doi.org/10.1007/s00103-020-03152-5

(c) Der/die Autor(en) 2020

Bundesverband Deutscher Krankenhausapotheker e. V. (ADKA) · Deutsche Gesellschaft für Hygiene und Mikrobiologie e.V. (DGHM) · Deutsche Gesellschaft für Infektiologie e. V. (DGI) · Deutsche Gesellschaft für Krankenhaushygiene e. V. (DGKH) · Deutsche Gesellschaft für Pädiatrische Infektiologie e. V. (DGPI) · Kommission Antiinfektiva, Resistenz und Therapie (Kommission ART) beim Robert Koch-Institut · Paul-EhrlichGesellschaft für Chemotherapie e. V. (PEG) · Robert Koch-Institut, Geschäftsstelle der Kommission ART

\section{Strukturelle und personelle Voraussetzungen für die Sicherung einer rationalen Antiinfektivaverordnung in Krankenhäusern. Positionspapier der Kommission Antiinfektiva, Resistenz und Therapie (Kommission ART) beim Robert Koch-Institut mit Beratung durch Fachgesellschaften}

\section{Zusammenfassung}

Gesteigertes Bewusstsein für die Problematik zunehmender Antibiotikaresistenzen und drohender Verlust geeigneter Therapieoptionen für Infektionen haben die Rahmenbedingungen in der Patientenversorgung verändert. In internationalen (WHO, ECDC) und nationalen Programmen und evidenzbasierten Leitlinien wurden Maßnahmen und Instrumente von Antibiotic Stewardship (ABS) konkretisiert. In Deutschland ist ABS im Krankenhaus im Infektionsschutzgesetz \$23 verankert und Empfehlungen dazu in einer S3-Leitlinie. Das vorliegende Positionspapier stellt Ziele von $A B S$ sowie erforderliche strukturelle und personelle Voraussetzungen für Krankenhäuser dar.

Für die erfolgreiche Arbeit des ABS-Teams sind die Sicherung einer qualifizierten Fort- und Weiterbildung, die Mandatierung, die Unterstützung und die Bereitstellung ausreichender Ressourcen durch die Krankenhausleitung unabdingbar. Das ABS-Team soll krankenhausweit und abteilungsübergreifend arbeiten. Aufgaben sind Ausarbeitung und Umsetzung eines auf die lokalen Bedürfnisse und Möglichkeiten des Krankenhauses zugeschnittenen ABSProgramms unter Berücksichtigung von Antiinfektivaeinsatz, Resistenzlage und Patientenschwerpunkten. Dazu kommen Erstellung und Implementierung hausinterner Empfehlungen zur Diagnostik, Prophylaxe und Therapie wichtiger Infektionen. Das ABSTeam soll interdisziplinär arbeiten und als Teammitglieder speziell qualifizierte Ärzte und Apotheker umfassen. ABS-beauftragte
Ärzte unterstützen das ABS-Team und berücksichtigen abteilungsspezifische Belange. Es wird von einer Mindestpersonalstärke für das $A B S$-Team von 1 Vollzeitäquivalent (VZÄ) pro 500 Betten ausgegangen. Je nach Krankenhausschwerpunkten und Anforderungen wird ein Zusatzbedarf gesehen. Die Wochenarbeitszeitstunden für einen ABS-beauftragten Arzt einer mittelgroßen Abteilung wurden auf 0,1 VZÄ pro 100 Betten geschätzt.

\section{Schlüsselwörter}

Antibiotic Stewardship · ABS-Team · Aufgaben des ABS-Teams · ABS-Personal · ABSPersonalbedarf

\section{Organisational and staff requirements for antimicrobial stewardship activities in hospitals. Position paper of the Commission on Anti-Infectives, Resistance and Therapy (Commission ART) at the Robert Koch Institute, with advice from professional associations}

\begin{abstract}
Increased awareness of the rising antimicrobial resistance problem and impending loss of suitable treatment options for infectious diseases have changed patient care. Antimicrobial/antibiotic stewardship (ABS) activities aiming to optimize antimicrobial treatment were specified in international (WHO, ECDC) and national programmes and evidence-based practice guidelines. In Germany, ABS in hospitals is enshrined in the Infection Protection Act $\$ 23$ and in a national guideline. The position paper presents the goals and tasks of ABS as well as the necessary organisational and staff requirements. Qualified training and education, mandates and support from hospital directors, and the
\end{abstract}

team and take the department-specific concerns into account. The document specifies a minimum ABS staff of one full-time equivalent (FTE) per 500 beds. Depending on the case mix and specialties, additional staff may be required. It proposes that there should be 0.1 FTE doctors commissioned for ABS per 100 beds.

Keywords

Antimicrobial stewardship - Antimicrobial stewardship team - Task of antimicrobial stewardship team · Human resources · Staffing requirements eigenständige Aufgabenfelder. Dazu gehören im Bereich ABS die Verbesserung der Infektionsdiagnostik und Antiinfektivabehandlungsqualität in Bezug auf Leitlinienadhärenz bei Wahl der Substanz, Dosis, Dauer, Therapiekontrolle, Eskalations- und Deeskalationsentschei- dungen sowie die Minimierung von sekundären Folgen des Antiinfektivaeinsatzes wie Resistenzentwicklung und Erregerselektion, aber auch unerwünschte Arzneimittelwirkungen, Toxizitäten und Interaktionsrisiken.
Die Verfügbarkeit von Erreger- und Resistenzdaten, die durch das betreuende mikrobiologische Labor erhoben werden, ist eine Voraussetzung für effektive ABS-Maßnahmen. Während Daten der Erreger- und Resistenz-Surveillance von der Hygiene genutzt werden, um die 
Infobox 1 Übersicht über Aufgaben und Funktionen des ABS-Teams

- Beauftragung durch die Krankenhausleitung mit der Planung und Implementierung eines krankenhausweiten ABS-Programms

- Planung und Ausarbeitung eines auf die hausinternen Bedürfnisse und die Situation des Krankenhauses zugeschnittenen ABS-Programms und von Strategien der Umsetzung in Kooperation mit den ABS-beauftragten Ärzten

- Organisation und regelmäßige Durchführung interner ABS-Teamtreffen mit Berichten

- Unterstützung der Integration von ABS in das Qualitätsmanagement des Krankenhauses; Festlegung von ABS-Qualitätsindikatoren und ggf. Zielbereichen

- Mitgliedschaft in Arzneimittelkommission und Hygienekommission, ggf. Infektionskommission

- Berücksichtigung der Anforderungen an Surveillance-Methoden zum Antiinfektivaverbrauch sowie zu Infektionserreger- und Resistenzstatistiken nach Infektionsschutzgesetz (IfSG), Mitgestaltung der krankenhausspezifischen Surveillance (interne und externe Qualitätssicherung) unter Berücksichtigung des hausinternen ABS-Programms und Bewertung sowie Kommunikation der Ergebnisse

- Planung und Durchführung von Punktprävalenzanalysen zu Antiinfektivaverordnungen und Adhärenz hausinterner Empfehlungen sowie Bewertung und Kommunikation der Ergebnisse

- Beteiligung bei der Erstellung und Implementierung hausinterner Empfehlungen und Behandlungspfade zur Diagnostik, Prophylaxe und Therapie der wichtigsten Infektionskrankheiten, basierend auf anerkannten internationalen oder nationalen Leitlinien unter Berücksichtigung der lokalen Epidemiologie und Gegebenheiten

- Beteiligung an der Ausarbeitung von hausinternen Empfehlungen zur Präanalytik mikrobiologischer Untersuchungen und Unterstützung bei deren Umsetzung; Beteiligung an der hausinternen Regelung zur Indikation notwendiger mikrobiologischer und laborchemischer Untersuchungen; Beteiligung an hausinternen Regelungen zur Mitteilung mikrobiologischer Befunde; Unterstützung bei der Interpretation mikrobiologischer Befunde

- Erstellung und Implementierung einer Antiinfektivahausliste, spezieller Sonderrezeptund Freigaberegelungen sowie Anwendungsbeschränkungen in Zusammenarbeit mit den Fachabteilungen

- Regelmäßige Durchführung von Fortbildungen/Schulungen/Informationsveranstaltungen zum Thema ABS

- Regelmäßig und bei Bedarf Durchführung von Antiinfektivavisiten mit den behandelnden Ärzten (sog. proaktive Verordnungsanalysen) auf Patientenebene (Visiten am Krankenbett oder Kurvenvisite)

- Bei Bedarf Absprache und Planung eines strategischen Wechsels von Substanzen oder Substanzklassen

Effektivität hygienischer und präventiver Interventionen zu überprüfen, dienen dieselben Daten zusammen mit Daten zum Antibiotikaverbrauch dazu, im Rahmen von ABS die Qualität des Antiinfektivaeinsatzes in Bezug auf Leitlinienadhärenz sowie Diagnose- und Behandlungsqualität zu bewerten und frühzeitig Resistenz- und Erregerselektionen zu erkennen. So kann eine sachgerechte Anpassung der infektiologischen Hausleitlinien und Behandlungspfade im Feedback mit den klinisch verantwortlichen Behandlungsteams erfolgen. Eine Unterstützung durch die IT-/EDV-Abteilung des jeweiligen Krankenhauses mit Bereitstellung von elektronischen Datensätzen $\mathrm{zu}$ Antiinfektivaverordnungen und Infektions- und Resistenzstatistiken, falls notwendig bis auf Patientenebene, ist eine wichtige Voraussetzung für die Planung, Umsetzung und Qualitätssicherung von ABS-Maßnahmen.
Das ABS-Team soll regelmäßig Treffen einberufen, die zu protokollieren und deren Protokolle zu archivieren sind. Es empfiehlt sich, ein Programm oder eine ABS-Strategie - gegebenenfalls mit quantitativen Zielen und unter Verwendung geeigneter Qualitätsindikatoren zu verabschieden. Das ABS-Team informiert die ABS-beauftragten Ärzte regelmäßig über geplante ABS-Maßnahmen sowie über Fortschritte und Probleme des ABS-Programms.

\section{Zusammensetzung des ABS-Teams und Qualifikation der Mitglieder}

Das ABS-Team soll interdisziplinär arbeiten und als Teammitglieder speziell qualifizierte Ärzte und Apotheker umfassen (siehe unten). Die Leitung und die Stellvertretung des ABS-Teams soll aus mindestens zwei im Krankenhaus beschäftigten Mitgliedern bestehen, darunter min- destens ein Arzt mit einer Facharztanerkennung in einem Gebiet der unmittelbaren Patientenversorgung. Die Leitung muss bei einem Arzt liegen.

Die Teammitglieder sollen nach Möglichkeit über infektionsmedizinische Qualifikationen gemäß den Weiterbildungsordnungen für Ärzte (Facharzt für innere Medizin und Infektiologie, Fachärzte mit Zusatz-Weiterbildung Infektiologie, Facharzt für Mikrobiologie, Virologie und Infektionsepidemiologie, Facharzt für Hygiene und Umweltmedizin und Apotheker (Bereichsweiterbildung Infektiologie)) verfügen. Die konkrete Zusammensetzung des ABS-Teams und die Wahl der Teamleitung richten sich nach hausinternen Gegebenheiten wie Krankenhausgröße, personelle Ausstattung, Spezialisierung u. a. Insbesondere in Krankenhäusern, in denen Fachärzte oder Apotheker mit infektionsmedizinischer Qualifikation nicht verfügbar sind, kann die Übertragung der Leitung des ABS-Teams an Fachärzte mit abgeschlossener Fortbildung zum ABS-Experten erfolgen.

Die Tätigkeit von interdisziplinären ABS-Teams gilt als kosteneffektiv und ist mit (nachhaltigen) positiven Wirkungen im Bereich Patientensicherheit und Resistenzminimierung verbunden. Die Einrichtung von ABS-Teams mit entsprechender Ausstattung wird bereits vielfach als Qualitätsindikator (Strukturqualität) für Akutkrankenhäuser betrachtet [27, 32].

\section{Aufgaben im ABS-Team je nach Qualifikation Klinisch tätiger Facharzt mit infektions- medizinischer Qualifikation. Facharzt} in einem Gebiet der unmittelbaren Patientenversorgung mit Zusatz-Weiterbildung Infektiologie, Facharzt für innere Medizin und Infektiologie oder übergangsweise ein klinisch tätiger Facharzt mit abgeschlossener Fortbildung zum ABS-Experten, zum Infektiologen (DGI) oder pädiatrischen Infektiologen (DGPI).

Klinisch tätige Fachärzte mit infektionsmedizinischer Qualifikation besitzen besondere Kenntnisse und Erfahrungen in der klinischen Diagnostik und Therapie komplexer Infektionen, in der speziellen Antiinfektivatherapie - gerade auch 
bei mehrfach resistenten Erregern - sowie der individuellen Prophylaxe, Verlaufsbeurteilung und Nachsorge, im Management von Infektionen bei Immundefekten, Fremdkörperinfektionen, importierten Infektionen, der Sepsis und des septischen Schocks. Ärzte mit der entsprechenden Qualifikation werden damit vor allem in größeren Krankenhäusern und in Krankenhäusern mit hohem Spezialisierungsgrad gebraucht für die Erstellung von evidenzbasierten, detaillierten hausinternen Empfehlungen, für Antiinfektivavisiten und für die sachgerechte Interpretation der Daten zum Antiinfektivaverbrauch sowie zur Mitarbeit bei der Interpretation von Infektionserreger- und Resistenzstatistiken. Sie sind besonders geschult und qualifiziert, durch infektiologische Konsiliartätigkeit sachgerechte Infektionsdiagnostik und rationale Antiinfektivaverordnung auch im komplexen Einzelfall zu sichern, die Umsetzung hausinterner Empfehlungen zu verbessern und ein direktes Feedback an die Kollegen der verschiedenen Fachabteilungen $\mathrm{zu}$ geben [33-38].

Sie können ihre Expertise beim Entwurf und der Konsentierung von hausinternen Empfehlungen einbringen und im Sinne dieses Positionspapiers die rationale Antiinfektivaverordnung auch am Krankenbett unterstützen. Sie können nach Absprache die regelmäßige Durchführung krankenhausinterner Fortbildungen, Schulungen und Informationsveranstaltungen organisieren und sollen die Möglichkeit erhalten, selbst spezifische Fortbildungsangebote wahrzunehmen (infektiologische Fortbildung und ABS-Refresher-Kurse). Die Fortbildung zum ABS-Experten soll in von den Landesärztekammern anerkannten Kursen, die 200 Unterrichtsstunden umfassen, in Anlehnung an das von der Deutschen Gesellschaft für Infektiologie (DGI) ausgearbeitete Curriculum erfolgen [39].

Mikrobiologe. Facharzt für Mikrobiologie, Virologie und Infektionsepidemiologie.

Mikrobiologen sind für die mikrobiologische Diagnostik zuständig und beraten Krankenhäuser in der sachgerechten Labordiagnostik von Infektionser- regern und bei der Antiinfektivatherapie. Sie unterstützen eine qualitativ hochwertige Infektionsmedizin durch gezielte Diagnostik, schnelle Befunderstellung und -übermittlung [40-42]. Ihre Expertise wird benötigt für die Erstellung von hausinternen Empfehlungen zur mikrobiologischen Präanalytik und Labordiagnostik sowie für die Gestaltung und Übermittlung mikrobiologischer Befunde nach nationalen und internationalen Qualitätsstandards [43]. Klinisch-mikrobiologische Visiten durch Mikrobiologen, die in der infektiologischen Konsiliartätigkeit erfahren sind, können den rationalen Einsatz von Antiinfektiva unterstützen [44]. Mikrobiologen und/oder Krankenhaushygieniker erstellen mit aktuellen und geprüften Daten eine auch für die Bewertung von ABS-Maßnahmen geeignete Infektionserreger- und Resistenzstatistik.

Krankenhaushygieniker. Facharzt für Hygiene und Umweltmedizin (oder Facharzt mit Zusatz-Weiterbildung Krankenhaushygiene oder mit abgeschlossener strukturierter curricularer Fortbildung Krankenhaushygiene).

Krankenhaushygieniker sind für die Prävention, Surveillance und Bekämpfung nosokomialer Infektionen und der Ausbreitung ihrer Erreger zuständig. Zu den Aufgaben des Hygienikers gehören auch die Prävention und Kontrolle der Verbreitung von Infektionserregern im belebten und unbelebten Umfeld (mittels Reinigung, Desinfektion, Sterilisation, Schutzmaßnahmen etc.), die Beratung der Krankenhausleitung und die Kommunikation von Hygienemaßnahmen an das medizinische Personal sowie die Koordination des Managements von Ausbrüchen. Die Ursachenanalyse von Infektionsausbrüchen ist eine zentrale Aufgabe des Hygienikers, zu ihr gehört auch die Bewertung von Herkunft und Verbreitungswegen der ausbruchsverantwortlichen Erreger. Bei Auftreten antibiotikaresistenter oder spezieller pathogener Erreger, z. B. C. difficile, ist die Kenntnis der Antibiotikaverbräuche und ihres Einflusses auf Erreger und Resistenzen essenziell.

Die Ziele von ABS ergänzen daher die Strategien zur Prävention von Antibioti- karesistenzen oder der Selektion pathogener Erreger, da auch sie der Kontrolle der Infektionserreger durch Minimierung der antibiotikaassoziierten Risiken dienen. ABS stellt damit eine wichtige Schnittstelle zu den präventiven Aufgaben der Krankenhaushygiene dar, auch wenn das Präventionspotenzial von ABS und Hygiene je nach Erreger unterschiedlich ist.

Fachapotheker. Fachapotheker mit Bereichsbezeichnung Infektiologie (oder Fachapotheker mit abgeschlossener Fortbildung zum ABS-Experten oder vertretungsweise Fachapotheker für klinische Pharmazie).

Fachapotheker verfügen über besondere pharmakotherapeutische Kenntnisse und bringen diese in Arzneimittelund Hygienekommissionen sowie in die Erstellung hausinterner Empfehlungen und Arzneimittellisten ein. Sie beraten die verordnenden Ärzte zur klinischen Relevanz unerwünschter Arzneimittelwirkungen, zu Interaktionen, zur Dosisoptimierung und -individualisierung. Sie sind für die Gestaltung, Umsetzung und Einhaltung der Antiinfektivahausliste mitverantwortlich. Die dafür erforderlichen speziellen Rezeptfreigabe- oder Sonderrezeptregelungen werden von Apothekern maßgeblich mitgestaltet [30, 44, 45]. Die Aufarbeitung von Arzneimittelverbrauchs- und Kostendaten für Surveillance- und Benchmarkzwecke gehört ebenfalls zu den Aufgaben des Apothekers. Sie bedienen sich dabei u. a. auch der Computerized-PhysicianOrder-Entry-Systeme (CPOE).

\section{ABS-beauftragte Ärzte an der Schnittstelle zwischen ABS-Team und Fachabteilung}

ABS-beauftragte Ärzte unterstützen das ABS-Team und stellen die Berücksichtigung abteilungsspezifischer Belange in der Antiinfektivatherapie und bei den ABS-Maßnahmen sicher. Die Befähigung zum ABS-beauftragten Arzt wird im Rahmen des 40-stündigen Grundkurses (Modul 1) des Curriculums Antibiotic Stewardship (ABS) der Bundesärztekammer erworben [39]. Diese Fortbildung kann bereits in der Weiterbildung zum 


\section{Infobox 2 Übersicht über Aufgaben und Funktionen des ABS-beauftragten Arztes}

- Beauftragter der klinischen Fachabteilung/ Fachabteilungsgruppe

- Teilnahme an den ABS-Teamsitzungen nach Bedarf (in der Geschäftsordnung geregelt)

- Mitwirkung bei der Kommunikation der Daten zu Antibiotikaverbrauch sowie zu Infektionserreger- und Resistenzstatistik in der Fachabteilung

- Mitarbeit bei der Durchführung von Punktprävalenzanalysen in der Fachabteilung

- Mitarbeit bei der Erstellung hausinterner Empfehlungen zur Diagnostik und Therapie fachgebietsrelevanter Infektionen und deren Implementierung sowie Vorstellung dieser Empfehlungen im Rahmen der fachabteilungsinternen Fortbildung

- Unterstützung bei der Umsetzung einer leitliniengerechten hausinternen Präanalytik und Ansprechpartner des mikrobiologischen Labors zur Sicherstellung einer leitliniengerechten Übermittlung mikrobiologischer Befunde

- Unterstützung bei der Umsetzung spezieller Sonderrezept-, Freigaberegelungen und Anwendungsbeschränkungen

- Patientenfallbasierte Schulungen im Rahmen der fachabteilungsinternen Fortbildung

- Teilnahme an Antiinfektivavisiten des ABS-Teams in der Fachabteilung

- Unterstützung bei fachbereichsbezogenen Ausbruchsuntersuchungen und eventuell notwendigen Änderungen diagnostischer Algorithmen und Antibiotikastrategien

Facharzt absolviert werden und wird für die Fortbildung zum ABS-Experten anerkannt. Die Herausforderungen der Antiinfektivatherapie in der Pädiatrie erfordern eine Fortbildungsmaßnahme mit spezifischen pädiatrischen Belangen.

Von den klinischen Fachbereichen als ABS-Beauftragte ernannte Ärzte arbeiten bei der Erstellung hausinterner Empfehlungen zur Diagnostik und Therapie fachgebiets- oder fachbereichsrelevanter Infektionskrankheiten mit und unterstützen die Kollegen der Fachabteilung bei der Implementierung hausinterner Empfehlungen auf den Stationen. Darüber hinaus stellen ABS-beauftragte Ärzte die hausinternen Empfehlungen in den Fachabteilungen bzw. -bereichen vor und sollen für fachbereichsinterne Schulungen zur Verfügung stehen. Die ABS- beauftragten Ärzte sollen regelmäßig spezifische Fortbildungen $\mathrm{zu}$ rationaler Antibiotikaverordnung und Infektionsdiagnostik anbieten. Ferner sollen sie ebenfalls die Möglichkeit erhalten, selbst entsprechende Fortbildungsangebote wahrzunehmen (infektiologische Fortbildung, Fortbildung in der Infektionsdiagnostik und ABS-RefresherKurse).

Eine Ernennung seitens der Fachabteilungen zum ABS-beauftragten und hygienebeauftragten Arzt (mit beiden Funktionen in Personalunion) kann bei entsprechender Qualifikation sinnvoll sein. ABS-beauftragte Ärzte sind Ansprechpartner des mikrobiologischen Labors zur Sicherstellung einer leitliniengerechten Präanalytik und Übermittlung mikrobiologischer Befunde und sollen den verantwortlichen Krankenhaushygieniker bei Ausbruchsuntersuchungen im Fachbereich (auf den Stationen) unterstützen. Eine Zusammenfassung der Aufgaben enthält • Infobox 2.

\section{Personalbedarf}

Die Personalbedarfsermittlung stützt sich auf Leitlinien und gesundheitspolitische Empfehlungen, Erfahrungen in der internationalen Literatur sowie auf Angaben zu den Grunddaten und Planungsgrundlagen für deutsche Krankenhäuser 2015 (Quellen: Statistisches Bundesamt bzw. Deutsche Krankenhausgesellschaft). Sie bezieht sich auf die Krankenversorgung und bezieht hier auch Krankenhäuser der Grund- und Regelversorgung sowie Schwerpunktkrankenhäuser mit ein, die im Rahmen der Krankenhausplanung mit den entsprechenden Ressourcen ausgestattet werden sollten, um entsprechende infektiologische Kompetenz in der Basisversorgung breiter aufzustellen. Insbesondere im Bereich der Universitätskrankenhäuser bzw. größerer akademischer Lehrkrankenhäuser der Tertiärversorgung ergibt sich zusätzlicher Bedarf durch Lehre und Forschung sowie Weiterbildungstätigkeit. Dieser Bedarf ist nicht Gegenstand des vorliegenden Positionspapiers und bedarf separater Berücksichtigung und Berechnung.

\section{ABS-Team}

Die deutsch-österreichische S3-Leitlinie hat die notwendige personelle Infrastruktur zur Durchführung von ABS-Programmen in Akutkrankenhäusern konkretisiert und empfiehlt eine Ausstattung von (mindestens) 1 Vollzeitstellenäquivalent (VZÄ) für ABSTeammitglieder pro 500 Betten, mit der ein Basisbündel von ABS-Maßnahmen in vielen Fällen kostenneutral durchführbar sein sollte [18]. Studien, die den Personalbedarf je nach Ausgangsposition und Zielerreichung bezüglich Antiinfektivaverordnungsqualität und Resistenzminimierung exakt quantifizieren können, sind bisher nicht verfügbar. Eine exakte Bezifferung des optimalen Personalbedarfs ist demnach erschwert, doch lassen sich aus Beschreibungen in der Literatur zum Personaleinsatz und aufgrund des geschätzten Zeitbedarfs (siehe Anhang) gemäß Aufgabenbeschreibung des ABS-Teams eine solche Mindestpersonalstärke und ein Zusatzbedarf je nach Krankenhausgröße und -struktur schätzen und begründen.

Es erscheint notwendig und wird angeregt, Kosteneffektivitätsstudien in Auftrag zu geben, die eine genauere Abschätzung der Investitionen in Personal und Infrastruktur unter Berücksichtigung des Versorgungsauftrags der Krankenhäuser erlauben und auch Möglichkeiten und Chancen regionaler Vernetzung in Deutschland aufzeigen.

\section{Basisbedarf: 1 Vollzeitstellenäqui- valent pro 500 Betten}

Nach verschiedenen Studien sind im Mittel mindestens 30\% der Antiinfektivaverordnungen in Akutkrankenhäusern optimierbar [46]. Bei einer durchschnittlichen Antibiotikabehandlungsinzidenz bei Krankenhauspatienten von $\sim 30 \%$, einem Anteil daran von $30 \%$ mit dringlichem Beratungsbedarf und einer mittleren Verweildauer von $\sim 1$ Woche bedeutet dies, dass pro 500 Betten ein individueller Beratungsbedarf in der Größenordnung von rund 40-50 Fällen pro Woche entsteht $(500 \times 0,3 \times 0,3=45)$. Dies entspricht wiederum den Ergebnissen eines jüngeren US-amerikanischen Surveys, in dem für Akutkrankenhäuser 
eine Rate von rund 10 Empfehlungen pro 100 Betten (und Woche) ermittelt wurde, um Fehler bezüglich Angemessenheit der Antibiotikabehandlung zu korrigieren oder $\mathrm{zu}$ vermeiden [47]. Alleinige apothekenbasierte Indikationsprüfungen können effektiv sein [48], jedoch eine ärztliche Beurteilung - optimalerweise fachärztlich-infektiologische Expertise mit Konsil am Krankenbett - nicht komplett ersetzen. Nimmt man einen Zeitbedarf von 30-60 min pro Beratungsfall an, ergeben sich $20-50 \mathrm{~h}$ pro Woche (0,5-1,25 VZÄ) bei einem 500Betten-Krankenhaus für infektiologische Konsile, ABS-Visiten und teilweise apothekenbasierte Indikationsprüfungen $(30 \mathrm{~min} \times 40$ Fälle $=20 \mathrm{~h}, 60 \mathrm{~min} \times 50$ Fälle $=50 \mathrm{~h}$, in der - Tab. 1 (im Anhang) wurden $25 \mathrm{~h}$ pro Woche angenommen). Hinzu kommen Aufgaben, die nicht direkt patientenbezogen sind, ABSTeamsitzungen/Mitarbeit in Gremien, Surveillance, Schulungen, Leitlinienarbeit etc.

Für kleinere Krankenhäuser (70-150 Betten) hat man kürzlich einen Zeitbedarf von insgesamt $5-10 \mathrm{~h}$ pro Woche (entsprechend rund $33 \mathrm{~h}$ pro Woche pro 500 Betten) ermittelt [49]. In einem jüngeren Konsensusreport aus den Niederlanden [50] wird angegeben, dass die Personalstärke - abhängig von der Krankenhausgröße und auch den angestrebten ABS-Zielen und -Maßnahmen - bei mindestens 0,87-1,1 (bei 300 Betten), 1,15-1,79 (bei 750 Betten) und 1,43-1,68 (bei 1200 Betten) VZÄ liegt. Dies entspricht einem Basisbedarf von 0,7-1,45 VZÄ pro 500 Betten - ähnlich also den Schätzungen von anderer Seite eines Mindestpersonalbedarfes von $\sim 1$ VZÄ pro 500 Betten. Bei Intensivierung des Programms mit zusätzlichen Zielsetzungen und Anforderungen sowie in der Initialphase der Implementierung ist der Bedarf höher [50]. In einem US-amerikanischen Survey wurde ein Bedarf von durchschnittlich 1,3 VZÄ (Ärzte und Apotheker) bei Krankenhäusern mit einer Bettenzahl von 100-300 ermittelt. Bei größeren Krankenhäusern war der durchschnittliche Bedarf entsprechend höher (1,6 VZÄ [300-500 Betten], 2,2 VZÄ [501-1000 Betten], 3,6 VZÄ [>1000 Betten]; [51]).
Dass sich mit einer Personalstärke in der Größenordnung 1 VZÄ pro 500 Betten ein Basisbündel von ABS-Maßnahmen in der Regel mindestens kostenneutral realisieren lässt, zeigen verschiedene Studien. Die bisher berichteten Kosteneinsparungen (Medikamente, Diagnostik) betrugen in einer jüngeren Literaturübersicht in den entsprechenden Studien zwischen 9000 US\$ und >2 Mio. US\$ pro Krankenhaus [52]. Es liegen auch Berichte über Nettoeinsparungen vor. So wurden am University of Maryland Medical Center (Baltimore, Maryland, USA) mit einer Personalausstattung von (umgerechnet) 1,5 VZÄ pro500 Betten Nettoeinsparungen (über Arzneimittelkosten, 500 US\$ pro Bett und Jahr, unter Berücksichtigung der Kosten des ABS-Programms) über 7 Jahre erzielt [53]. In einem deutschen Universitätskrankenhaus wurden über 2 Jahre Nettoeinsparungen (ebenfalls nur Arzneimittelausgaben betreffend, $500 €$ pro Bett und Jahr) mit einem Personaleinsatz von $2 \mathrm{VZÄ} \mathrm{pro}$ 500 Betten erzielt [54]. In einer kürzlich publizierten Studie [31] war eine Investition in Fachpersonal in dieser Höhe auch in einem schwedischen Krankenhaus für den Krankenhausträger mindestens kostenneutral (Reduktion der Arzneimittelausgaben inkl. Pflegepersonalzeit - ohne Berücksichtigung der Erlöseffekte durch kürzere Liegedauern und erhöhte Fallzahlen). In einer niederländischen Studie wurden im Rahmen der Tätigkeit eines ABS-Teams innerhalb eines Jahres Nettoeinsparungen mit hoher Rentabilität (Vollkosten für eine Beratung/Konsil $\sim 80 €$, Einsparung $~ 500 €$ pro Fall durch verkürzte Verweildauer, weniger Krankenpflegeaufwand, geringere Arzneimittelkosten) erzielt [28].

Das vorliegende Positionspapier geht entsprechend der obigen Angaben ebenfalls von einer begründbaren Mindestpersonalstärke für das ABS-Team in der Größenordnung von 1 VZÄ pro 500 Betten aus. Dies ist im Einklang mit den Empfehlungen der europäischen Gesundheitsbehörden (ECDC), die für die Durchführung kosteneffektiver ABSProgramme und unter Verweis auf die notwendige Sicherung einer optimierten Versorgungs- bzw. Verordnungsqualität 1-3 VZÄ pro 500 Betten fordern [27].
Der über 1 VZÄ hinausgehende Bedarf pro 500 Betten wurde dort vor allem aufgrund eines französischen Surveys aufgenommen, der einerseits neben Infektiologen weitere Disziplinen im ABSTeam berücksichtigt und außerdem auch einen Zusatzbedarf bei größeren Krankenhäusern mit Spezialdisziplinen wiedergibt (siehe unten).

Bei der Berechnung der Mindestpersonalstärke sollen für die zugrunde $\mathrm{zu}$ legende Bettenzahl psychiatrische, psychotherapeutische/psychosomatische, nuklearmedizinische und Rehabilitationsbetten (außer Akutrehabilitation) nicht mitgezählt werden.

Gerade in kleinen Krankenhäusern wird sich ein eigenständiges ABS-Team je nach den lokalen Gegebenheiten aus einem oder mehreren infektiologisch qualifizierten Krankenhausärzten, dem vor Ort angestellten Krankenhaushygieniker, der gleichzeitig ABS-Experte sein kann, sowie je nach Präsenz einem infektiologisch qualifizierten Pharmazeuten und einem beratenden Mikrobiologen, die ebenfalls ABS-fortgebildet sein sollten, zusammensetzen.

Die Doppelqualifikation Infektiologe/ABS-Experte und Krankenhaushygieniker sollte gefördert, die jeweiligen Stellenanteile gemäß Größe des Krankenhauses addiert werden. Alternativ könnte der errechnete ABS-Stellenanteil auch auf mehrere ABS-qualifizierte Personen verteilt werden.

\section{Zusatzbedarf bis zu 0,5 Vollzeit- stellenäquivalente bei bestimmten Krankenhausschwerpunkten}

Neben der Mindestpersonalstärke wird je nach Krankenhausschwerpunkten (komplexitätsbedingt) und Anforderungen ein Zusatzbedarf gesehen. Hierzu gibt es keine systematischen Untersuchungen. Diese Sichtweise stützt sich aber auf Erfahrungswerte in deutschen Krankenhäusern und entspricht im Wesentlichen auch den bisherigen internationalen Erfahrungen und auch den Empfehlungen der ECDC und anderer Institutionen. So wurde in Frankreich früher als Mindestpersonalstruktur eine Rate von 0,375 VZÄ pro 500 Betten („référent d'antibiotiques") angegeben [55], jedoch aus der Praxis mit Hinweis auf einen we- 
sentlich höheren Bedarf (insgesamt 3,3 VZÄ pro 500 Betten) korrigiert [56]. Bezogen auf 500 Betten wurden dort für die einzelnen Fachdisziplinen im ABS-Team folgende Bedarfszahlen (VZÄ) genannt: $\sim 1,8$ Infektiologen, $\sim 1,25$ Apotheker, $\sim 0,3$ Mikrobiologen/Hygieniker [56]. Ein über 1 VZÄ pro 500 Betten hinausgehender Bedarf findet sich auch in der australischen ABS-Empfehlung (2 VZÄ pro 500 Betten; [57]), in einer früheren US-amerikanischen Studie ( 1,5 VZÄ für mittelgroße Krankenhäuser; [58]) sowie auch in den neuen Vorgaben der US-amerikanischen Centers for Medicare \& Medicaid Services zu neuen Qualitätsstandards für Krankenhäuser ( 1,4 VZ̈̈ pro 500 Betten; [59]). Die oben genannte niederländische Arbeitsgruppe schätzte den Bedarf für ein intensiviertes Programm auf (umgerechnet) 1,3-2,1 VZÄ pro 500 Betten [50].

In den Beratungen zum vorliegenden Positionspapier wurde mehrheitlich dafür gestimmt, dass folgende Krankenhausmerkmale und Schwerpunkte einen Zusatzpersonalbedarf für das ABS-Team von je 0,5 VZÄ definieren sollen - unabhängig von der Zahl der für diese Schwerpunkte vorgehaltenen Betten:

- Krankenhäuser mit Organtransplantationszentrum bzw. -einheiten,

- Krankenhäuser mit einem Schwerpunkt in der Hämatologie inkl. allogener Stammzelltransplantation,

- Krankenhäuser mit Fachabteilung für Kinder- und Jugendmedizin inkl. Neonatologie,

- Krankenhäuser mit eigenen Schwerpunkten im Bereich orthopädischer Gelenkersatz und/oder Herzchirurgie und/oder Neurochirurgie (wenn nur eine $(0,2$ VZÄ) oder $2(0,4$ VZÄ) der Disziplinen vorhanden sind, entsprechende Reduktion),

- Krankenhäuser mit mehr als 4 intensivmedizinischen Behandlungseinheiten/Stationen oder mehr als 50 Intensivbetten oder einer eigenen (bettenführenden) Fachabteilung Intensivmedizin.

- Tab. 2 zeigt beispielhaft die Berechnung des Personalbedarfs für ein fachabteilungsübergreifendes ABS-Team.
Die exakte Aufteilung der Aufgaben und Stundenzahlen muss und kann nur unter Berücksichtigung der Verfügbarkeit von Fachpersonal vor Ort, der konkreten Struktur der klinischen Schwerpunkte und im Einvernehmen mit der ABS-Teamleitung definiert werden. $\mathrm{Zu}$ berücksichtigen sind bei den Stellenanteilen für Hygieniker die Empfehlungen der Kommission für Krankenhaushygiene und Infektionsprävention (KRINKO) zu personellen Voraussetzungen für eine sachgerechte Krankenhaushygiene [60, 61].

Dem ABS-Team ggf. (zeitlich begrenzt) zugeordnete Ärzte in Weiterbildung in den einschlägigen infektionsmedizinischen Fächern übernehmen im Rahmen ihrer Weiterbildung Aufgaben in der Versorgung und sind daher in die Stellenkalkulation einzubeziehen. Dabei kann in den ersten beiden Jahren der Weiterbildung die Kapazität des Weiterzubildenden mit $50 \%$ des Beschäftigungsumfanges und $a b$ dem 3. Jahr der Weiterbildung mit $90 \%$ des Beschäftigungsumfanges angerechnet werden.

\section{ABS-beauftragte Ärzte}

In Abhängigkeit von Krankenhausgröße und Spezialisierungsgrad der klinischen Fachabteilungen sollten zusätzlich sogenannte ABS-beauftragte Ärzte in den klinischen Fachabteilungen bzw. für definierte Fachabteilungen oder Fachabteilungsgruppen ernannt werden, um bei der Erstellung und Umsetzung lokaler Empfehlungen zur Diagnostik, Therapie und Prophylaxe von Infektionen die Belange spezieller Fächer zu berücksichtigen [18]. Die Beschreibung dieser Verantwortlichkeiten und Aufgaben ist in Analogie zur vorgeschlagenen Krankenhaushygienestruktur $\mathrm{zu}$ verstehen (Krankenhaushygieneteam mit Facharzt bzw. Krankenhaushygieniker - abteilungsübergreifend - plus hygienebeauftragte Ärzte pro klinischer Fachabteilung als Ansprechpartner und in die Hygienekommission delegierte Ärzte). Hygieneund ABS-beauftragte Ärzte der Fachabteilungen können diese Funktionen in Personalunion wahrnehmen.
Alle krankenhausinternen Fachabteilungen mit unmittelbarer Patientenversorgung sowie die intensivmedizinischen Versorgungbereiche, die über eine eigene ärztliche Leitung verfügen, sollen jeweils einen ABS-beauftragten Arzt benennen (außer Psychiatrie, Psychotherapie/Psychosomatik, Nuklearmedizin). Fachabteilungsgruppen (z. B. innere Medizin, Chirurgie) können zusammengelegt werden, wenn sie gemeinsam eine Bettenzahl von 100 nicht überschreiten und dies fachlich als adäquat bewertet wird. Belegarztabteilungen sowie kleine Abteilungen ( $<15$ Betten) können grundsätzlich durch ABS-beauftragte Ärzte anderer Fachabteilungen mit unmittelbarer Patientenversorgung mitbetreut werden.

Die Wochenarbeitszeitstunden für einen ABS-beauftragten Arzt einer mittelgroßen bettenführenden Abteilung (40-80 Betten) wurden von der Autorengruppe des Positionspapiers geschätzt. Sie dürften hochgerechnet etwa 0,1 VZÄ pro 100 Betten betragen, aufgrund der Komplexität bestimmter Schwerpunkte auch mehr. Dies ist bei Freistellungen bzw. Stellenplänen zu berücksichtigen.

\section{Korrespondenzadresse}

Robert Koch-Institut, Geschäftsstelle der

\section{Kommission ART}

Geschäftsstelle der Kommission ART 37

„Nosokomiale Infektionen, Surveillance von

Antibiotikaresistenz und -verbrauch, Abteilung für Infektionsepidemiologie", Robert KochInstitut

Seestr. 10, 13353 Berlin, Deutschland Kommission-ART@RKI.de

Kommission ART. Prof. Dr. Dr. rer. nat. Marianne Abele-Horn; Dr. Dr. Katja de With; Prof. Dr. Julia Seifert (ehemaliges Mitglied)

PEG. Prof. Dr. Dr. rer. nat. Marianne Abele-Horn

RKI. Dr. Tim Eckmanns, Dr. Nicole Schmidt

ADKA. Dr. Matthias Fellhauer

DGHM. Prof. Dr. Georg Häcker

DGI. Prof. Dr. Winfried Kern

DGPI. Prof. Dr. Johannes G. Liese

DGKH. Dr. Peter Walger

Funding. Open Access funding provided by Projekt DEAL. 


\section{Einhaltung ethischer Richtlinien}

Interessenkonflikt. Dieses Positionspapier wurde ehrenamtlich und ohne Einflussnahme kommerzieller Interessengruppen im Auftrag der Kommission Antiinfektiva, Resistenz und Therapie erarbeitet von Prof Dr. Dr. rer. nat. Marianne Abele-Horn, Dr. Dr. Katja de With, Dr. Matthias Fellhauer, Prof. Dr. Georg Häcker, Prof. Dr. Winfried Kern, Prof. Dr. Johannes G. Liese, Prof. Dr. Julia Seifert und Dr. Peter Walger. Vom RKI waren beteiligt: Dr. Tim Eckmanns und Dr. Nicole Schmidt. Das Positionspapier wurde durch die Autorengruppe vorbereitet und nach ausführlicher Diskussion in der Kommission abgestimmt.

Für diesen Beitrag wurden von den Autoren keine Studien an Menschen oder Tieren durchgeführt. Für die aufgeführten Studien gelten die jeweils dort angegebenen ethischen Richtlinien.

Open Access. Dieser Artikel wird unter der Creative Commons Namensnennung 4.0 International Lizenz veröffentlicht, welche die Nutzung, Vervielfältigung, Bearbeitung, Verbreitung und Wiedergabe in jeglichem Medium und Format erlaubt, sofern Sie den/die ursprünglichen Autor(en) und die Quelle ordnungsgemäß nennen, einen Link zur Creative Commons Lizenz beifügen und angeben, ob Änderungen vorgenommen wurden.

Die in diesem Artikel enthaltenen Bilder und sonstiges Drittmaterial unterliegen ebenfalls der genannten Creative Commons Lizenz, sofern sich aus der Abbildungslegende nichts anderes ergibt. Sofern das betreffende Material nicht unter der genannten Creative Commons Lizenz steht und die betreffende Handlung nicht nach gesetzlichen Vorschriften erlaubt ist, ist für die oben aufgeführten Weiterverwendungen des Materials die Einwilligung des jeweiligen Rechteinhabers einzuholen.

Weitere Details zur Lizenz entnehmen Sie bitte der Lizenzinformation auf http://creativecommons.org/ licenses/by/4.0/deed.de. 


\section{Anhang}

Tab. 1 Abschätzung des Personalbedarfs im ABS-Team (Ärzte, Apotheker), exemplarisch für ein 500-Betten-Krankenhaus mit 25 Stationen bei 10 Fachabteilungen pro Jahr ohne Berücksichtigung besonderer Schwerpunkte mit erhöhter Patientenkomplexität (Schätzung der Autorengruppe)

Tätigkeit

Stunden, Frequenz

Stundenberechnung

Hochrechnung aufs Jahr (Gesamtstunden)

ABS-Teamsitzungen

Punktprävalenzerhebungen zu Antiinfektivaverordnungen inkl. Auswertung und Bericht/Kommunikation der Ergebnisse

Regelmäßige ABS-Visiten und teilweise apothekenbasierte Indikationsprüfungen, Beratungen (Konsile)

Leitlinienerstellung und Aktualisierung je nach Fachabteilung

Fortbildung/Schulung, Kommunikation und Feedback je nach Fachabteilung

Erreger-/Resistenz- sowie Antibiotikaverbrauchsstatistikinkl. Auswertung und Kommunikation

Planung, Durchführung, Supervision von ABS-Projekten zur Therapieoptimierung (z. B. Präanalytik mikrobiologischer Diagnostik, Oralisierung)

Zusätzliche Personalstunden ergeben sich aus Aufgaben im Kontext des Qualitätsmanagements, für Mitarbeit in Hygiene- und Arzneimittelkommission, Gesundheitsamtsbegehungen, Ausbruchsmanagement, Netzwerkarbeit, Supervision der ABS-beauftragten Ärzte, Berichtspflicht gegenüber der Krankenhausleitung etc.

\begin{tabular}{|l|l|l|}
\hline- & - & $\begin{array}{l}\text { Gesamtstunden pro } \\
\text { Jahr ohne Urlaub/ } \\
\text { Fortbildung }\end{array}$ \\
\hline- & - & $\begin{array}{l}\text { Gesamtstunden }(+15 \% \\
\text { wg. Urlaub/Fortbildung })\end{array}$ \\
\hline $\mathrm{a}=40 \mathrm{~h}$ pro Woche & $2075^{\mathrm{a}}$ \\
\hline
\end{tabular}

\section{$8 \mathrm{~h}$ pro Quartal}

$4 \mathrm{~h}$ pro Erhebung (einmal pro Jahr pro Fachabteilung) und Auswertung $+4 \mathrm{~h}$ pro Bericht/ Kommunikation

$1 \mathrm{~h}$ pro Visite pro Woche pro Station

$8 \mathrm{~h}$ pro Jahr pro Fachabteilung

$3 \mathrm{~h}$ pro Quartal pro Fachabteilung inkl. Vorbereitung

\section{$16 \mathrm{~h}$ pro Quartal}

$16 \mathrm{~h}$ pro Quartal

16h pro Quartal

\section{2}

$4 \times 2 \times 10$

80

$25 \times 52$

1300

$8 \times 10$

80

$3 \times 4 \times 10$ 120

$16 \times 4$

64

$16 \times 4$

64

$16 \times 4$

Tab. 2 Beispiel für Personalbedarfsberechnung ABS-Team

Krankenhaustyp/-größe ${ }^{a}$ und Schwerpunkte

Universitätskrankenhaus 1200 Betten mit Organtransplantationseinheit, Hämatologie inkl. allogener Stammzelltransplantation, mit Fachabteilung für Kinder- und Jugendmedizin inkl. Neonatologie und eigenen Fachabteilungen für Herzchirurgie und für Neurochirurgie, 5 Intensivstationen

Großes Allgemeinkrankenhaus

Betten mit Fachabteilung für Kinder- und Jugendmedizin inkl. Neonatologie und eigenen Fachabteilungen für Neurochirurgie, Herzchirurgie, und Orthopädie mit Schwerpunkt Gelenkersatz (nach obiger Definition)

Mittleres Allgemeinkrankenhaus

450 Betten mit Orthopädie (Gelenkersatz) als Schwerpunkt (nach obiger Definition)

Kleines Allgemeinkrankenhaus

250 Betten ohne Schwerpunkte

\section{Mindestbedarf}

(1 VZÄ pro 500

Betten)

2,4

Zusatzbedarf (0,5 VZÄ je definie

Gesamt-

2,5

(VZÄ)

4,9

0,9

0,2

1,1

0,5

0,5

${ }^{a}$ Ohne Berücksichtigung von psychiatrischen, psychotherapeutischen/psychosomatischen, nuklearmedizinischen und Rehabilitationsbetten 


\section{Literatur}

1. National Institute for Health and Care Excellence (2015) Antimicrobial stewardship: systems and processes for effective antimicrobial medicine use, S50

2. Agriculture AGDoHa (2015) Australia's First National Antimicrobial Resistance Strategy 2015-2019. https://www.agriculture.gov.au/animal/health/ amr/antimicrobial-resistance-strategy. Zugegriffen:26. März 2020

3. Health Protection Surveillance Centre (HPSC) AR (2012) Health Protection Surveillance Centre (HPSC), Annual Report 2012. http://www. hpsc.ie/A-Z/Gastroenteric/Clostridiumdifficile/ CdifficileSurveillance/AnnualReports/File, 14557, en.pdf.Zugegriffen: 26. März 2020

4. Barlam TF, Cosgrove SE, Abbo LM et al (2016) Implementing an antibiotic stewardship program: guidelines by the infectious diseases Society of America and the Society for Healthcare Epidemiology of America. Clin Infect Dis 62:e51-e77

5. House TW (2015) National action plan for combating antibiotic-resistant bacteria. https://www. cdc.gov/drugresistance/pdf/national_action_ plan_for_combating_antibotic-resistant_ bacteria.pdf. Zugegriffen: 26. März 2020

6. Ministère des Solidarités et dela Santé(Hrsg) (2016) Ministère du travail deedls Plan National d'Alerte sur les Antibiotiques 2011-2016. http://socialsante.gouv.fr/IMG/pdf/plan_antibiotiques_20112016 DEFINITIF.pdf.Zugegriffen: 26. März 2020

7. SDWPoA (2015) Practical guide antimicrobial stewardship in the Netherlands. http:// esgap.escmid.org/wp-content/uploads/2015/11/ SWAB_guideline_ABS_hospitals.pdf.Zugegriffen: 26. März 2020

8. de With K, Kern WV, Strauß R, Kramme E, Friedrichs $\mathrm{A}$, Holzmann T, Geiss HK, Isner C, Fellhauer $\mathrm{M}$ von Ameln-Mayerhofer A, AbeleHorn M, Häcker G, Walger P, Deja M, Vehreschild JJ, Kather A, Friese E, Porsche U, Janata O, Krause R, Wechsler-Fördös A (2018) S3-Leitlinie Strategien zur Sicherung rationaler Antibiotika-Anwendung im Krankenhaus. In: AWMF online, S88

9. S2kLeitlinie „Antibiotic Stewardship-Konzeption und Umsetzung in der stationären Kinder- und Jugendmedizin“ In:Deutsche Gesellschaft für Pädiatrische Infektiologie (DGPI)

10. de With K, Allerberger F, Amann S et al (2016) Strategies to enhance rational use of antibiotics in hospital: a guideline by the German Society for Infectious Diseases. Infection 44(3):395-439. https://doi.org/10.1007/s15010-016-0885-z

11. Simon A, von Both U, Hufnagel $M$, Hübner J (2018) Antibiotic Stewardship in der Kinderund Jugendmedizin. Monatsschr Kinderheilkd 166:48-56

12. KernWV, deWith K(2012) Rationale Antibiotikaverordnung. Bundesgesundheitsblatt 55:1418-1426

13. Kern WV (2018) Rationale Antibiotikaverordnung in der Humanmedizin. Bundesgesundheitsblatt 61:580-588

14. Kern WV (2012) Fortbildungsinitiative Antibiotic Stewardshipfür Krankenhausärzte und-apotheker. Krankenhhyg up2date 7:47-54

15. Walger PFG, Herrmann M, Liese J, Mertens T (2017) Infektionsmedizin: Klinische Expertise fördern. Dtsch Arztebl 114:A734-A735

16. Kern WV, Fätkenheuer G, Tacconelli E, Ullmann A (2015) Klinische Infektiologie in Deutschland und Europa. Z Evid Fortbild Qual Gesundhwes 109:493-499. https://doi.org/10.1016/j. zefq.2015.09.015
17. Schweickert B, Kern WV, de With Ket al (2013) Surveillance of antibiotic consumption : Clarification of the "definition of data on the nature and extent of antibiotic consumption in hospitals according to section sign 23 paragraph 4 sentence 2 of the IfSG" Bundesgesundheitsblatt Gesundheitsforschung Gesundheitsschutz 56:903-912

18. AWMF, AdWMF (2019) S3-Leitlinie 092/001 - Strategien zur Sicherung rationaler Antibiotika-Anwendung im Krankenhaus, update 2018. https:// www.awmf.org/uploads/tx_szleitlinien/092 001I_S3_Strategien-zur-Sicherung-rationalerAntibiotika-Anwendung-im-Krankenhaus_201904.pdf.Zugegriffen:26. März 2020

19. NICE (2015) Guideline antimicrobial stewardship - systems and processes for effective antimicrobial medicine use. https://www.nice.org.uk/ guidance/ng15.Zugegriffen:26. März 2020

20. Centre HPS (2009) Guidelines for antimicrobial stewardship in hospitals in Ireland. https://www.hpsc ie/A-Z/MicrobiologyAntimicrobialResistance/ InfectionControlandHAl/Guidelines/File,4116, en.pdf.Zugegriffen:26. März 2020

21. MacDougall C, Polk RE (2005) Antimicrobial stewardship programs in health care systems. Clin Microbiol Rev 18:638-656

22. Davey P, Marwick CA, Scott CL et al (2017) Interventions to improve antibiotic prescribing practices for hospital inpatients. Cochrane Database Syst Rev. https://doi.org/10.1002/14651858. CD003543.pub4

23. Karanika S, Paudel S, Grigoras C, Kalbasi A, Mylonakis E (2016) Systematic review and metaanalysis of clinical and economic outcomes from the implementation of hospital-based antimicrobial stewardship programs. Antimicrob Agents Chemother 60:4840-4852

24. Schuts EC, Hulscher ME, Mouton JW et al (2016) Current evidence on hospital antimicrobia stewardship objectives: a systematic review and meta-analysis. Lancet Infect Dis 16:847-856

25. Lawes T, Lopez-Lozano JM, Nebot CA et al (2015) Effects of national antibiotic stewardship and infection control strategies on hospital-associated and community-associated meticillin-resistant Staphylococcus aureus infections across a region of Scotland: a non-linear time-series study. Lance Infect Dis 15:1438-1449

26. Lawes T, Lopez-Lozano JM, Nebot CA et al (2017) Effect of a national $4 C$ antibiotic stewardship intervention on the clinical and molecular epidemiology of Clostridium difficile infections in a region of Scotland: a non-linear time-series analysis. Lancet Infect Dis 17:194-206

27. ECDC (2017) ECDC TECHNICAL REPORT Proposals for EU guidelines on the prudent use of antimicrobials in humans. http://ecdc.europa. eu/en/publications/Publications/EU-guidelinesprudent-use-antimicrobials.pdf. Zugegriffen: 26 März2020

28. Dik JW, Hendrix R, Friedrich AW et al (2015) Cost-minimization model of a multidisciplinary antibiotic stewardship team based on a successful implementation on a urology ward of an academic hospital. PLoSONE 10:e126106

29. Goff DA, File TM Jr. (2016) The evolving role of antimicrobial stewardship in management of multidrug resistant infections. Infect Dis Clin North Am 30:539-551

30. Brink AJ, Messina AP, Feldman C et al (2016) Antimicrobial stewardship across 47 South African hospitals: an implementation study. Lancet Infect Dis 16:1017-1025
31. Lanbeck P, Ragnarson Tennvall G, Resman F (2016) A cost analysis of introducing an infectious disease specialist-guided antimicrobial stewardship in an area with relatively low prevalence of antimicrobial resistance. BMC Health Serv Res 16:311

32. Pollack LA, Plachouras D, Sinkowitz-Cochran $R$ Gruhler H, Monnet DL, Weber JT (2016) A concise set of structure and process indicators to assess and compare antimicrobial stewardship programs among EU and US hospitals: results from a multinational expert panel. Infect Control Hosp Epidemiol 37:1201-1211

33. Chou AF, Graber CJ, Jones $M$ et al (2016) Characteristics of antimicrobial stewardship programs at veterans affairs hospitals: results of a nationwide survey. InfectControl HospEpidemiol 37:647-654

34. Fawcett NJ, Jones N, Quan TP et al (2016) Antibiotic use and clinical outcomes in the acute setting under management by an infectious diseases acute physician versus other clinical teams: a cohort study. BMJOpen 6:e10969

35. Lesprit $P$, de Pontfarcy $A$, Esposito-Farese $M$ et al (2015) Postprescription review improves in-hospital antibiotic use: a multicenter randomized controlled trial. Clin Microbiol Infect 21:180.e181-180.e187

36. Pulcini C, Botelho-Nevers E, Dyar OJ, Harbarth S (2014) The impact of infectious disease specialists on antibioticprescribing in hospitals. Clin Microbiol Infect 20:963-972

37. Rieg S, Küpper MF (2016) Infectious diseases consultations can make the difference: a brief review and a plea for more infectious diseases specialists in Germany. Infection 44:159-166

38. Rieg S, Hitzenbichler F, Hagel S et al (2019) Infectious disease services: a survey from four university hospitals in Germany. Infection 47:27-33

39. Bundesärztekammer (2017) Curriculum „Antibiotic Stewardship (ABS)“ Rationale Antiinfektivastrategien im Krankenhaus

40. Messacar K, Parker SK, Todd JK, Dominguez SR (2017) Implementation of rapid molecular infectious disease diagnostics: the role of diagnostic and antimicrobial stewardship. J Clin Microbiol 55:715-723

41. Morency-Potvin P, Schwartz DN, Weinstein RA (2017) Antimicrobial stewardship: how the microbiology laboratory can right the ship. Clin Microbiol Rev 30:381-407

42. Timbrook TT, Morton JB, McConeghy KW, Caffrey AR, Mylonakis E, LaPlante KL (2017) The effect of molecular rapid diagnostic testing on clinical outcomes in bloodstream infections: a systematic review and meta-analysis. Clin Infect Dis 64:15-23

43. Johnson LS, Patel D, King EA, Maslow JN (2016) Impact of microbiology cascade reporting on antibiotic de-escalation in cefazolin-susceptible Gram-negative bacteremia. Eur J Clin Microbiol Infect Dis 35:1151-1157

44. Kim J, Craft DW, Katzman M (2015) Building an antimicrobial stewardship program: cooperative roles for pharmacists, infectious diseases specialists, and clinical microbiologists. Lab Med 46:e65-e71

45. Gilchrist M, Wade P, Ashiru-Oredope D et al (2015) Antimicrobial stewardship from policy to practice: experiences from UK antimicrobial pharmacists. Infect Dis Ther 4:51-64

46. Marquet K, Liesenborgs A, Bergs J, Vleugels A, Claes N (2015) Incidence and outcome of inappropriate in-hospital empiric antibiotics for severe infection: a systematic review and metaanalysis. Crit Care 19:63 


\section{Positionspapier}

47. Livorsi DJ, Heintz B, Jacob JT, Krein SL, Morgan DJ, Perencevich EN (2016) Audit and feedback processes among antimicrobial stewardship programs: a survey of the society for healthcare epidemiology of america research network. Infect Control Hosp Epidemiol 37:704-706

48. CaoH, PheK, Laine GA, Russo HR, Putney KS, Tam VH (2016) An institutional review of antimicrobial stewardship interventions. J Glob Antimicrob Resist 6:75-77

49. Stenehjem E, Hyun DY, Septimus E et al (2017) Antibiotic stewardship in small hospitals: barriers and potential solutions. Clin Infect Dis 65:691-696

50. Ten Oever J, Harmsen M, Schouten J et al (2018) Human resources required for antimicrobial stewardship teams: a Dutch consensus report. Clin Microbiol Infect 24:1273-1279

51. Doernberg SB, Abbo LM, Burdette SD et al (2018) Essential resources and strategies for antibiotic stewardship programs in the acute care setting Clin Infect Dis 67:1168-1174

52. Nathwani D, Varghese D, Stephens J, Ansari W, Martin S, Charbonneau C (2019) Value of hospital antimicrobial stewardship programs [ASPs]: a systematic review. Antimicrob Resist Infect Control 8:35

53. Standiford HC, Chan S, Tripoli M, Weekes E, Forrest GN (2012) Antimicrobial stewardship at a large tertiary care academic medical center: cost analysis before, during, and after a 7-year program. Infect Control Hosp Epidemiol 33:338-345

54. Borde JP, Kaier K, Steib-Bauert M et al (2014) Feasibility and impact of an intensified antibiotic stewardship programme targeting cephalosporin and fluoroquinolone use in a tertiary care university medical center. BMC Infect Dis 14:201

55. SantéMdASedl (2012) Direction Générale del'Offre de Soins-Instruction noDGOS/PF2/2012/286 du 16 juillet 2012.http://social-sante.gouv.fr/fichiers/ bo/2012/12-08/ste_20120008_0100_0042.pdf. Zugegriffen: 26. März 2020

56. Le Coz P, Carlet J, Roblot F, Pulcini C (2016) Human resources needed to perform antimicrobial stewardship teams' activities in French hospitals. Med Mal Infect 46:200-206

57. Duguid MCM (2011) Antimicrobial stewardship in Australian hospitals. https:// www.safetyandquality.gov.au/wp-content/ uploads/2011/01/Antimicrobial-stewardship-inAustralian-Hospitals-2011.pdf. Zugegriffen: 26. März 2020

58. Owens RC Jr., Fraser GL, Stogsdill P (2004) Antimicrobial stewardship programs as a means to optimize antimicrobial use. Insights from the Society of Infectious Diseases Pharmacists. Pharmacotherapy 24:896-908

59. Medicare and Medicaid Programs; Hospital and Critical Access Hospital (CAH) (2016) Changes to promote innovation, flexibility, and improvement in patient care; proposed rule. https://www.federalregister.gov/ documents/2016/06/16/2016-13925/medicareand-medicaid-programs-hospital-and-criticalaccess-hospital-cah-changes-to-promote. Zugegriffen:26. März 2020

60. KRINKO (2009) Personelle und organisatorische Voraussetzungen zur Prävention nosokomialer Infektionen. Bundesgesundheitsblatt Gesundheitsforschung Gesundheitsschutz 52:951-962

61. KRINKO (2016) Empfehlung zum Kapazitätsumfang für die Betreuung von Krankenhäusern und anderen medizinischen Einrichtungen durch Krankenhaushygieniker/innen. Bundesgesundheitsblatt Gesundheitsforschung Gesundheitsschutz 59:1183-1188 